\title{
Artificial neural networks for the definition of kinetic subpopulations in electroejaculated and epididymal spermatozoa in the domestic cat
}

\author{
Alberto Contri, Daniele Zambelli ${ }^{1}$, Massimo Faustini ${ }^{2}$, Marco Cunto ${ }^{1}$, Alessia Gloria \\ and Augusto Carluccio
}

Department of Veterinary Clinical Sciences, University of Teramo, Viale Crispi 212, 64100 Teramo, Italy, ${ }^{1}$ Department of Veterinary Medical Sciences, Animal Reproduction Unit, University of Bologna, Via Tolara di Sopra 50, 40064 Ozzano dell'Emilia, Bologna, Italy and ${ }^{2}$ Department of Veterinary Sciences and Public Health, Università degli Studi di Milano, Via Celoria 10, 20133 Milan, Italy

Correspondence should be addressed to A Contri; Email: acontri@unite.it

\begin{abstract}
This study was designed for the identification of different sperm kinetic subpopulations in feline semen using artificial neural networks (ANNs) and for the evaluation of the effect of ejaculation on motility patterns of these subpopulations. Seven tomcats presented for routine orchiectomy were electroejaculated, and after 5 days, orchiectomized and epididymal tail sperms were collected. Sperm motility characteristics were evaluated using a computer-assisted sperm analyzer that provided individual kinetic characteristics of each spermatozoon. A total of 23400 spermatozoa for electroejaculated and 9200 for epididymal tail samples were evaluated using a multivariate approach, comprising principal component analysis and ANN classification. The multivariate approach allowed the identification and characterization of three different and well-defined sperm subpopulations. There were significant differences before (epididymal tail spermatozoa) and after (electroejaculated sperm) ejaculation in sperm kinetic subpopulation characteristics. In both epididymal and ejaculated samples, the majority of subpopulation was characterized by high velocity and progressiveness; however, the electroejaculated samples showed significantly higher values, suggesting that the microenvironment of the epididymal tail could affect the sperm motility or, alternatively, seminal plasma could increase the kinetic characteristics of the spermatozoa, indicating that only after ejaculation, the spermatozoa express their motility potential. Nevertheless, further studies are required to clarify the functional significance of each kinetic subpopulation.
\end{abstract}

Reproduction (2012) 144 339-347

\section{Introduction}

Feline semen evaluation is basically performed for commercial, diagnostic, and research purposes. Several wild species of felids are considered threatened or endangered, and the domestic cat can be considered as an excellent experimental model for research in those felids (Zambelli \& Cunto 2006).

Several studies have highlighted, in the raw ejaculate, the presence of different subpopulations classified based on specific characteristics, such as morphology (NunezMartinez et al. 2005), osmotic resistance (Perez-Llano et al. 2003, Quintero-Moreno et al. 2004), and acrosome status (Perez-Llano et al. 2003). However, the sperm kinetic parameters are the most-used features to identify sperm subpopulations in humans (Chantler et al. 2004) and animals (Holt et al. 1996, Abaigar et al. 1999, 2001, Quintero-Moreno et al. 2003, 2004, 2007, MartinezPastor et al. 2005a, Nunez-Martinez et al. 2006).
Motility estimation is an essential step in the evaluation of male fertility, because it can be considered as a functional test; it is a direct determination of the energy status of the mammalian sperm (QuinteroMoreno et al. 2004) because most of the energy that spermatozoa produce is involved in motility (Roldan 1998). Development of computer-assisted sperm analyzers (CASA) has allowed the objective evaluation of sperm motility, also giving a numerical quantity for several kinetic characteristics. These devices have afforded identification of spermatozoa across several successive digitalized microscopic images, thus providing their trajectories. Hence, CASA systems can supply velocity and kinetic parameters of hundreds or thousands of spermatozoa. However, this amount of information has so far been underrated, and motility evaluation has been limited to the classical univariate statistical analysis, variable by variable. This restricted overview could diminish the whole information value 
or trigger erroneous conclusions (Abaigar et al. 1999). The use of the mean value is the result of the simplistic assumption that spermatozoa parameters follow a normal distribution. However, some studies have revealed a complex structure of sperm population with respect to their kinetic characteristics using multivariate approach to CASA-derived data (Abaigar et al. 1999, Quintero-Moreno et al. 2003, Chantler et al. 2004, Nunez-Martinez et al. 2006, Martinez-Pastor et al. 2011).

The artificial neural network (ANN) is a biologically inspired computational model developed to simulate the way in which human brain processes data. It consists of networks of highly interconnected virtual neurons that can accept input features and produce an output decision on the basis of its 'experience.' Thus, the ANN learns by a specific training process and makes a decision on the basis of this experience (Fogel 2008). This flexibility is used in medicine to make diagnosis, verify the usefulness of a treatment, or predict the outcome of complex clinical situations with many clinical, biological, and pathological variables because of their ability to exploit the intricate relationship between these variables. Furthermore, the ANNs are used to classify and recognize patterns accurately (Ramesh et al. 2004).

The aim of this study was to identify different sperm kinetic patterns in feline semen using multivariate statistics and ANN classification for data exploration and mining. As motility function takes place in the epididymis and to evaluate the possible effect of accessory sexual gland secretions on sperm kinetic, we also tried to verify the difference in sperm motility patterns between epididymal and ejaculated spermatozoa.

\section{Results}

The mean volume of electroejaculated semen was $62.5 \pm 45 \mu \mathrm{l}$ and the concentration was $215 \pm 124$ $\times 10^{6} \mathrm{sperm} / \mathrm{ml}$. The semen characteristics of the electroejaculated and epididymal samples are listed in Table 1. Data on the sperm kinetic characteristics reported (Table 1) were increased from a total of 23400 and 9200 sperm tracks in electroejaculated and epididymal samples respectively. The difference in the amount of the tracks evaluated was due to the epididymal collection, during which spermatozoa was diluted in a greater volume of medium. All the sperm kinetic parameters were far from normality (AndersonDarling test, $P \leq 0.01$ ) in the electroejaculated as well as epididymal sperms.

The wide ranges in these characteristics did not reveal significant differences, thus a multivariate approach was used. Principal component analysis (PCA) allowed individuation and classification of the principal components (PCs) based on their importance; thus, the first three populations comprised about $80 \%$ sperm variability and were characterized by different weights of each original parameter. Each PC was described by different kinetic parameters; thus, PC1 was strongly characterized by the dance (DANCE), curvilinear velocity $(\mathrm{VCL})$, amplitude of lateral head displacement $(\mathrm{ALH})$, average path velocity (VAP), and straight-line velocity (VSL) in both electroejaculated and epididymal spermatozoa. With the exception of VSL for PC1 and $\mathrm{PC} 2$, none of the sperm kinetic characteristics were observed in the PCs considered (Fig. 1).

Thus, each of the 23400 electroejaculated and 9200 epididymal spermatozoa was classified according to the weight of its PCs by the ANN methods. This procedure

Table 1 Summary of feline semen and kinetic characteristics, as median, 25th and 75th percentile, for electroejaculated and epididymal spermatozoa.

\begin{tabular}{|c|c|c|c|c|c|c|c|c|}
\hline & \multicolumn{4}{|c|}{ Electroejaculated } & \multicolumn{4}{|c|}{ Epididymal } \\
\hline & Anderson-Darling & 25 th & Median & 75th & Anderson-Darling & 25 th & Median & 75th \\
\hline $\operatorname{VAP}(\mu \mathrm{m} / \mathrm{s})$ & $<0.01$ & 32.9 & $119^{\mathrm{A}}$ & 175.1 & $<0.01$ & 23.7 & $88.5^{\mathrm{B}}$ & 144.7 \\
\hline $\operatorname{VSL}(\mu \mathrm{m} / \mathrm{s})$ & $<0.01$ & 16.3 & $86^{\mathrm{A}}$ & 146.5 & $<0.01$ & 11.7 & $60.8^{\mathrm{B}}$ & 121.9 \\
\hline $\mathrm{VCL}(\mu \mathrm{m} / \mathrm{s})$ & $<0.01$ & 91.3 & $183.7^{\mathrm{A}}$ & 252.7 & $<0.01$ & 67.9 & $165.8^{\mathrm{B}}$ & 242.9 \\
\hline $\mathrm{ALH}(\mu \mathrm{m})$ & $<0.01$ & 4.1 & $5.9^{\mathrm{A}}$ & 7.9 & $<0.01$ & 3.7 & $5.9^{\mathrm{A}}$ & 8.1 \\
\hline $\mathrm{BCF}(\mathrm{Hz})$ & $<0.01$ & 35.6 & $45^{\mathrm{A}}$ & 53.3 & $<0.01$ & 34.3 & $46.2^{\mathrm{A}}$ & 55.7 \\
\hline STR $(\%)$ & $<0.01$ & 55.0 & $78^{\mathrm{A}}$ & 92.0 & $<0.01$ & 56.0 & $79^{\mathrm{A}}$ & 91.0 \\
\hline LIN (\%) & $<0.01$ & 22.0 & $45^{\mathrm{A}}$ & 64.0 & $<0.01$ & 21.0 & $40^{\mathrm{B}}$ & 54.0 \\
\hline ELONG (\%) & $<0.01$ & 51.0 & $62^{\mathrm{A}}$ & 80.0 & $<0.01$ & 52.0 & $71^{\mathrm{B}}$ & 98.0 \\
\hline SIZE $\left(\mu \mathrm{m}^{2}\right)$ & $<0.01$ & 3.9 & $4.9^{\mathrm{A}}$ & 6.1 & $<0.01$ & 2.5 & $4.9^{\mathrm{A}}$ & 6.5 \\
\hline WOBBLE (\%) & $<0.01$ & 41.4 & $60.2^{\mathrm{A}}$ & 73.9 & $<0.01$ & 37.9 & $51.7^{\mathrm{B}}$ & 62.5 \\
\hline DANCE $\left(\mu \mathrm{m}^{2} / \mathrm{s}\right)$ & $<0.01$ & 426.3 & $1043.2^{\mathrm{A}}$ & 1846.9 & $<0.01$ & 268.9 & $954.2^{\mathrm{B}}$ & 1917.2 \\
\hline MeDANCE (\%) & $<0.01$ & 0.08 & $0.14^{\mathrm{A}}$ & 0.26 & $<0.01$ & 0.09 & $0.2^{\mathrm{B}}$ & 0.3 \\
\hline Head defects ${ }^{a}$ & - & 0 & $0.97^{\mathrm{A}}$ & 3.17 & - & 0 & $1.99^{\mathrm{A}}$ & 9.02 \\
\hline Midpiece defects ${ }^{\mathrm{a}}$ & - & 0 & $0.4^{\mathrm{A}}$ & 0.97 & - & 0 & $0.4^{\mathrm{A}}$ & 0.88 \\
\hline Tail defects ${ }^{\mathrm{a}}$ & - & 5.8 & $18.5^{\mathrm{A}}$ & 35.3 & - & 0 & $12.8^{\mathrm{A}}$ & 30.6 \\
\hline Acrosomal defects $\mathrm{a}^{\mathrm{a}}$ & - & 3.7 & $4.4^{\mathrm{A}}$ & 5.2 & - & 0 & $6.7^{\mathrm{A}}$ & 13.4 \\
\hline
\end{tabular}

In the same row, medians with different superscripted letters differ significantly $(P \leq 0.05$, Kruskal-Wallis/Steel-Dwass test). VAP, average path velocity; VSL, straight line velocity; VCL, curvilinear velocity; ALH, amplitude of lateral head displacement; BCF, beat cross frequency; STR, straightness;

LIN, linearity; ELONG, head elongation; SIZE, head size; WOB, Wobble's coefficient; DANCE, the dance; MeDANCE, mean dance.

aThe '25th' and the ' $75 \mathrm{th}^{\prime}$ ' columns represent the minimum and maximum respectively. 

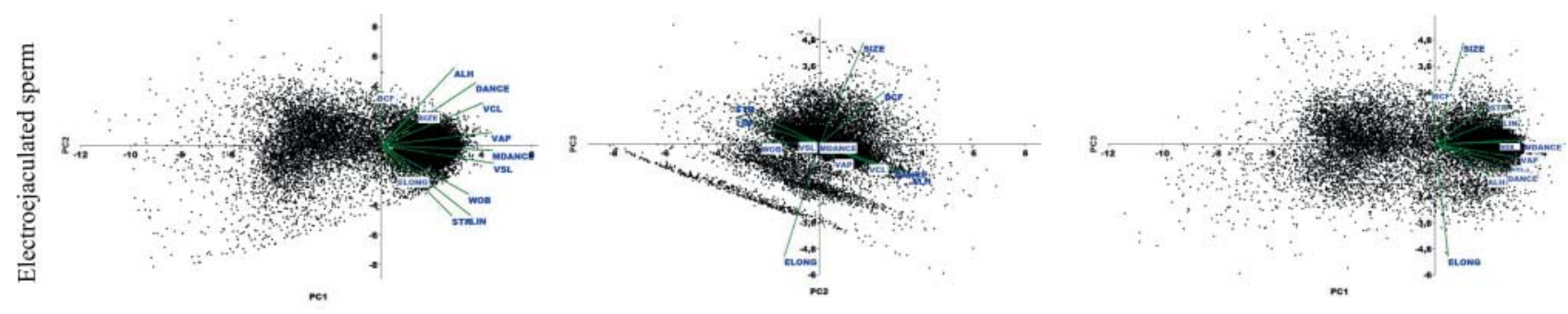

$\mathrm{PC} 1 \times \mathrm{PC} 2$

$\mathrm{PC} 2 \times \mathrm{PC} 3$

$\mathrm{PCl} \times \mathrm{PC} 3$

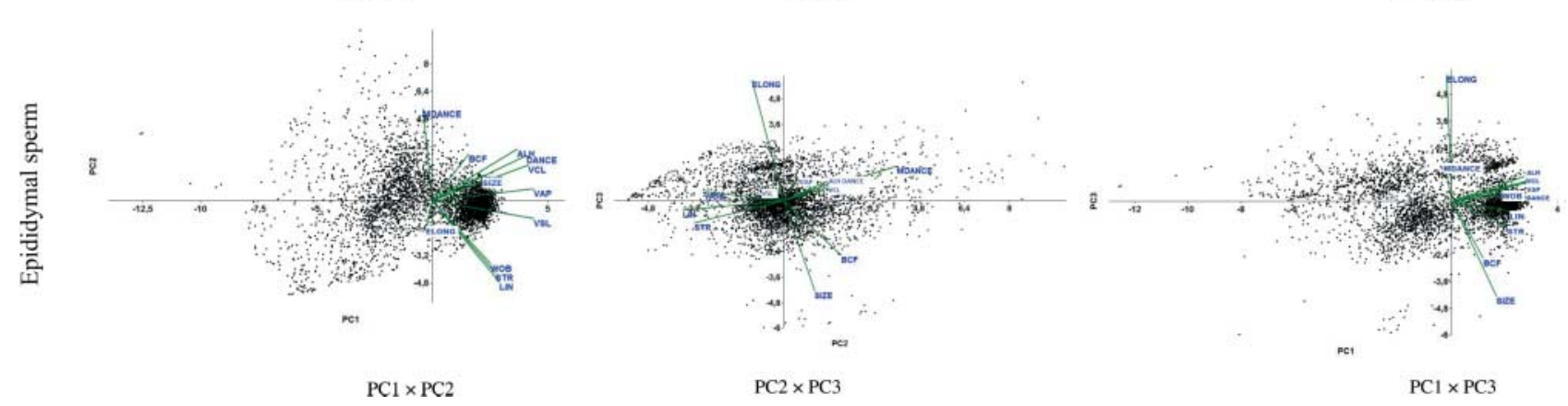

Figure 1 Biplots of the principal component (PC) analysis for the first three PCs considered (PC1 vs PC2, PC2 vs PC3, and PC1 vs PC3) in the electroejaculated and epididymal feline spermatozoa.

allowed hypothesizing the presence of three clusters of spermatozoa as different kinetic sperm subpopulations characterized by specific values of the kinetic parameters. The choice of three clusters was made by observing the kernel densities of the spermatozoa scores projected on the plane of the three variables (Fig. 2).

As shown in Table 2, cluster 1 in electroejaculated semen was characterized by relatively high VCL and low $\mathrm{VAP}$, indicating nonlinear trajectories of several spermatozoa. This finding was confirmed by low straightness (STR), linearity (LIN), and Wobble's coefficient (WOB), as well as by high amplitude and frequency of the head movement (ALH and beat cross frequency (BCF)). On the other hand, different values were noticed in epididymal spermatozoa (Table 3), in which this cluster had very low velocity (low VAP, VSL, and VCL) but relatively high progressiveness (high STR, LIN, and WOB). Cluster 2 was more numerous in both electroejaculated and epididymal semen $(68.26$ and $63.73 \%$ of the sperm population respectively) and was characterized by a linear high velocity (high VAP, VSL, and VCL) and progressive (high STR and LIN) motion (Tables 2 and 3). In cluster 3, spermatozoa were quite vigorous (relatively high VAP, VSL, and VCL) and progressive (high STR, LIN, and WOB) in electroejaculated samples but not (low STR, LIN, and WOB) in epididymal ones (Tables 2 and 3). Evaluation of the kinetic parameters of the subpopulation in electroejaculated and epididymal tail spermatozoa compared using Welch-corrected $t$-test for independent groups found that electroejaculated (Table 2) and epididymal spermatozoa (Table 3) were statistically different on the basis of their subpopulations; however, some of the observed differences could have emerged owing to the high sample size.

Significant differences were present among toms in both electroejaculated and epididymal samples. There were some differences in the subpopulation distribution between the toms. Cluster 2 was the most represented in Tom 1, 2, 4, and 7, while in Tom 3, 5, and 6, the percentage of spermatozoa with sperm kinetic characteristics of clusters 1 and 3 was higher.

\section{Discussion}

Some studies have attempted to relate motion parameters as well as in vivo fertility and IVF in both humans and animals using average value and parametric statistical approach (Holt et al. 1985, 1997, Marshburn et al. 1992, Barratt et al. 1993, Krause 1995, MacLeod \& Irvine 1995). However, this approach considers a normal distribution of all variables, while semen is made up of billions of individual spermatozoa that make this regular distribution unlikely. A previous study on boar semen highlighted the need for a different approach apart from the classical parametric and nonparametric analyses (Abaigar et al. 1999). Recently, different multivariate approaches for sperm motility evaluation were proposed (Abaigar et al. 1999, 2001, Quintero-Moreno et al. 2003, 2004, 2007, Martinez-Pastor et al. 2005a, Nunez-Martinez et al. 2006). Irrespective of the statistical procedure used, the aim of multivariate analysis in our study was to consider a global evaluation of all spermatozoa and whole motility parameters by simplifying the number of individuals and descriptors 

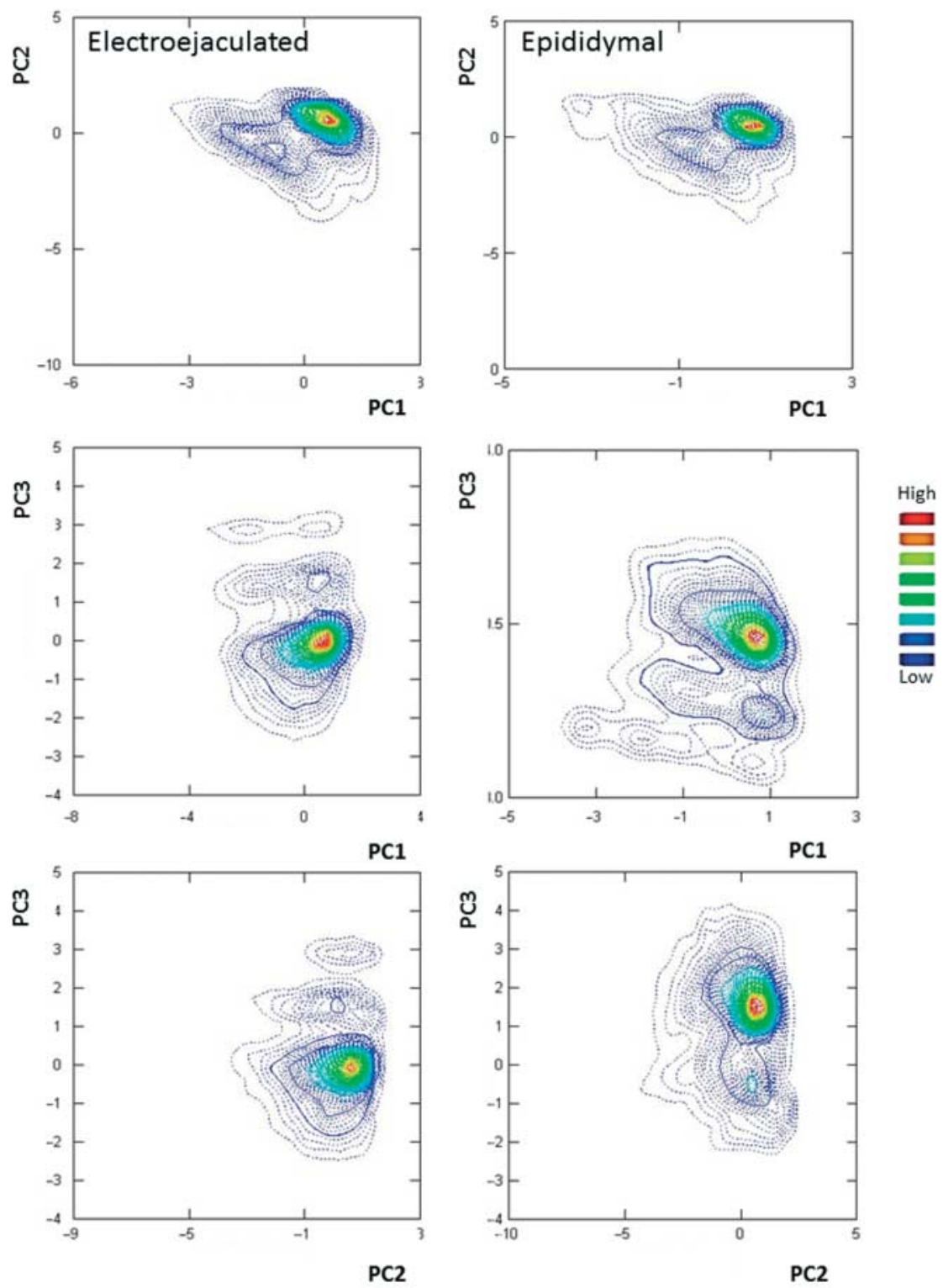

Figure 2 Kernel densities for the electroejaculated (left column) and epididymal (right column) spermatozoa reported on the principal component (PC) planes. The color indicates the arbitrary density (scale on the right).

without losing their information content (Abaigar et al. 1999). On the other hand, PCA allowed a good simplification using three variables (PCs) that explain about $80 \%$ of the total variability. Similar results were reported in several studies, in which $73 \%$ (QuinteroMoreno et al. 2003), more than $80 \%$ (Martinez-Pastor et al. 2005a), and 91\% (Nunez-Martinez et al. 2006) of variances were achieved.

Unlike several papers that achieved the identification of different subpopulations by the use of one or more mathematical procedures (Abaigar et al. 1999, 2001, Quintero-Moreno et al. 2003, 2004, 2007, MartinezPastor et al. 2005a, Nunez-Martinez et al. 2006), in this study, a neural network classification was used for the identification of kinetic characteristics of each subpopulation. The ANN is a collective name of an ensemble of mathematical techniques aimed to mimic the functions of the animal brain in a simple way. It consists of networks of highly interconnected 'neurons' that are capable of performing parallel computations for data processing and knowledge representation (Ramesh et al. 2004). As in real neuronal networks, an artificial neuron belonging to a network forms 'synapses' with other neurons: the connections between neurons are weighted and their intensities are defined by a function (generally sigmoidal, linear, or discrete) and an activation threshold (McCulloch \& Pitts 1943).

The advantages of the ANN are the plasticity of the procedures and its capacity to 'learn' and become self-experienced (Cartwright 2008) on the basis of a logical process, similar to the functioning of the brain (Agatonovic-Kustrin \& Beresford 2000). A neural 
Table 2 Summary for the median, 25th, and 75th percentile of kinetic characteristics for each subpopulation in electroejaculated feline sperm. For each cluster, the proportion (\%) on the whole spermatozoa was reported.

\begin{tabular}{|c|c|c|c|c|c|c|c|c|c|}
\hline \multirow[b]{2}{*}{ Variable } & \multicolumn{3}{|c|}{ Cluster $1(12.46 \%)$} & \multicolumn{3}{|c|}{ Cluster $2(68.26 \%)$} & \multicolumn{3}{|c|}{ Cluster $3(19.28 \%)$} \\
\hline & 25th & Median & 75th & 25th & Median & 75th & 25th & Median & 75th \\
\hline $\operatorname{VAP}(\mu \mathrm{m} / \mathrm{s})$ & 25.5 & 34 & 55.6 & 70.3 & 143.9 & 189.7 & 15.2 & 24.4 & 128.8 \\
\hline VSL $(\mu \mathrm{m} / \mathrm{s})$ & 6.7 & 10 & 16.9 & 55.5 & 115.5 & 160.4 & 9.2 & 16.8 & 100.8 \\
\hline $\operatorname{VCL}(\mu \mathrm{m} / \mathrm{s})$ & 83.55 & 110.1 & 159 & 139.5 & 214.1 & 265.3 & 37 & 57.1 & 195.1 \\
\hline $\operatorname{ALH}(\mu \mathrm{m})$ & 5 & 6.4 & 8.6 & 4.7 & 6.2 & 8.1 & 1.6 & 2.7 & 5.4 \\
\hline $\mathrm{BCF}(\mathrm{Hz})$ & 43.2 & 55.4 & 60 & 37.8 & 45 & 52.5 & 0.1 & 33.9 & 50.8 \\
\hline STR (\%) & 19 & 29 & 38 & 67 & 84 & 93 & 58 & 76 & 92 \\
\hline LIN (\%) & 7 & 10 & 13 & 34 & 53 & 68 & 23 & 36 & 62 \\
\hline ELONG $(\%)$ & 44 & 60 & 77 & 50 & 62 & 75 & 60 & 95 & 100 \\
\hline SIZE $\left(\mu \mathrm{m}^{2}\right)$ & 3.2 & 4.4 & 5.8 & 4.5 & 5.4 & 6.5 & 0.8 & 2.5 & 4.2 \\
\hline WOBBLE (\%) & 27.7 & 33.4 & 43.2 & 51.2 & 65.6 & 76.1 & 38.7 & 52.2 & 71.9 \\
\hline DANCE $\left(\mu \mathrm{m}^{2} / \mathrm{s}\right)$ & 436.9 & 694.9 & 1319 & 680.3 & 1315.8 & 2039.2 & 63.2 & 159.1 & 987.6 \\
\hline MeDANCE (\%) & 0.5 & 0.7 & 1 & 0.1 & 0.1 & 0.2 & 0.05 & 0.09 & 0.15 \\
\hline
\end{tabular}

For all variables, all cluster medians were pairwise different with $P<0.0001$ (Kruskal-Wallis test). Average path velocity (VAP), straight-line velocity (VSL), curvilinear velocity (VCL), amplitude of lateral head displacement (ALH), beat cross frequency (BCF), straightness (STR), linearity (LIN), head elongation (ELONG), head size (SIZE), Wobble's coefficient (WOB), the dance (DANCE), and mean dance (MeDANCE).

network 'learns' through repeated adjustments of these weights.

Several papers have reported the use of ANN in medicine, mainly for the diagnosis and prognostic evaluation of several pathologies (for a review, Ramesh et al. (2004)). Samli \& Dogan (2004) as well as Ma et al. (2011) reported the use of ANN as a predictive tool in human andrology. To our knowledge, this study is the first to use this technique for the clusterization of data, with the aim to analyze the subpopulation motility architecture in human and animal sperms.

The double-step classification of spermatozoa parameters (PCA and neural network classifier) allowed global evaluation of all spermatozoa (23 400 and 9200 for electroejaculated and epididymal sperms respectively) and detection of the existence of three distinct populations of spermatozoa based on the 12 parameters considered by IVOS, both in the electroejaculated and in the epididymal feline samples. This finding was in agreement with a previous study on boar semen, in which three sperm motility subpopulations were found (Abaigar et al. 1999), confirming the ability of the neural network classification to cluster sperm subpopulation. However, comparison of values revealed strong difference in sperm kinetic characteristics of each subpopulation, which must be due to different analytical criteria (mathematical vs logical) used for the statistical analysis. Furthermore, the technical characteristics of the CASA systems used were different, affecting the motility value of each spermatozoon. The CASA used in this study was equipped with illumination strobed at 1/1000 of a second to visualize sperm motion. This strobed illumination eliminated motion-related blurring along the length of the sperm head, resulting in precise sperm tracking. Furthermore, the image capture rate of $60 \mathrm{frames} / \mathrm{s}$ and 45 frames per field (a total of ten fields for a sample) provided high level of accuracy for measuring sperm velocities and motion parameters. Previous reports on equine (Quintero-Moreno et al. 2003), boar (Abaigar et al. 1999), deer (Martinez-Pastor et al. 2005a), dog

Table 3 Summary for the median, 25th, and 75th percentile of kinetic characteristics for each subpopulation in epididimal feline sperm. For each cluster, the proportion (\%) on the whole spermatozoa was reported.

\begin{tabular}{|c|c|c|c|c|c|c|c|c|c|}
\hline \multirow[b]{2}{*}{ Variable } & \multicolumn{3}{|c|}{ Cluster $1(14.47 \%)$} & \multicolumn{3}{|c|}{ Cluster $2(63.72 \%)$} & \multicolumn{3}{|c|}{ Cluster $3(21.81 \%)$} \\
\hline & 25th & Median & 75th & 25th & Median & 75th & 25th & Median & 75th \\
\hline $\operatorname{VAP}(\mu \mathrm{m} / \mathrm{s})$ & 5.8 & 10.6 & 14.1 & 49.1 & 122.3 & 154.3 & 27.3 & 54.3 & 124.8 \\
\hline $\operatorname{VSL}(\mu \mathrm{m} / \mathrm{s})$ & 3 & 7.4 & 10.2 & 32 & 100.4 & 134.8 & 9 & 20.5 & 87.6 \\
\hline $\operatorname{VCL}(\mu \mathrm{m} / \mathrm{s})$ & 11 & 24.7 & 36.4 & 114.5 & 203.1 & 259.6 & 85.3 & 143.7 & 224.3 \\
\hline $\mathrm{ALH}(\mu \mathrm{m})$ & 0.6 & 1.2 & 1.7 & 4.8 & 6.5 & 8.5 & 4.6 & 6.5 & 8.6 \\
\hline $\mathrm{BCF}(\mathrm{Hz})$ & 0.01 & 30 & 57.4 & 39.2 & 48 & 55.2 & 24 & 45 & 60 \\
\hline STR (\%) & 55 & 72.5 & 92 & 67 & 84 & 92 & 27 & 48 & 83 \\
\hline LIN (\%) & 19 & 29.5 & 51 & 29 & 46 & 55 & 9 & 18 & 44 \\
\hline ELONG (\%) & 51 & 100 & 100 & 50 & 63 & 78 & 76 & 100 & 100 \\
\hline $\operatorname{SIZE}\left(\mu \mathrm{m}^{2}\right)$ & 0.8 & 0.8 & 3.5 & 4.7 & 5.8 & 7.1 & 0.8 & 0.8 & 3.9 \\
\hline WOBBLE (\%) & 34.1 & 44.7 & 65.7 & 43.7 & 54.6 & 63.4 & 30.8 & 42.7 & 57.4 \\
\hline DANCE $\left(\mu \mathrm{m}^{2} / \mathrm{s}\right)$ & 7.1 & 29.8 & 62.4 & 595.9 & 1273.2 & 2132.8 & 428.6 & 931.3 & 1850 \\
\hline MeDANCE (\%) & 0.01 & 0.04 & 0.08 & 0.1 & 0.16 & 0.23 & 0.16 & 0.36 & 0.72 \\
\hline
\end{tabular}

For all variables all cluster medians were pairwise different with $P<0.001$ (Kruskal-Wallis/Steel Dwass test). VAP, average path velocity; VSL, straight line velocity; VCL, curvilinear velocity; ALH, amplitude of lateral head displacement; BCF, beat cross frequency; STR, straightness; LIN, linearity; ELONG, head elongation; SIZE, head size; WOB, Wobble's coefficient; DANCE, the dance; MeDANCE, mean dance. 
(Nunez-Martinez et al. 2006), and rabbit (QuinteroMoreno et al. 2007) sperm subpopulations used a frame rate of $25 \mathrm{~Hz}$ for image acquisition. Furthermore, different studies on the effects of technical settings on canine (Rijsselaere et al. 2003) and bovine (Contri et al. 2010) motility parameters demonstrated different results of mean sperm characteristics, recorded using the same device and the same sample but with different frame rates. In addition, significant increase in VAP, VSL, VCL, $\mathrm{BCF}$, LIN, and STR, but a reduction in ALH value, was found using a frame rate of $60 \mathrm{~Hz}$ (Mortimer \& Swan 1995, Rijsselaere et al. 2003, Contri et al. 2010).

In this study, a majority of the sperm population (cluster 2) of both electroejaculated and epididymal semen was characterized by a fast and progressive motility pattern (Tables 2 and 3). The 'fast and linear' subpopulation has been proposed as a good indicator of sample quality, whereas a predominant 'slow and nonlinear' subpopulation has been determined to be a marker of poor quality (Martinez-Pastor et al. 2005b, 2011). In a study on boar sperm subpopulation motility, no correlation was found between kinetic values of different subpopulations and fertility (Quintero-Moreno et al. 2003). However, in that study, the majority of the sperm subpopulation (about 90\%) was characterized by low velocity and high progressiveness, while the rest were found to have a very active but nonlinear motility. These differences in the motility pattern of the subpopulations could explain the inability to correlate fertility results to the characteristics of the sperm subpopulation. Spermatozoa with low or altered movement hardly reach the oviduct, and it is reasonable to consider that the higher the number of spermatozoa with progressive motility, the greater is the chance for one of them to reach the ampulla of the oviduct (Muino et al. 2008). In our study, it was not possible to correlate the size and kinetic characteristics of the major subpopulation to the fertility of the male because fertility trials should be performed on several females, hardly reached in feline reproduction.

The presence of different sperm subpopulations could be interpreted as different functional and physiological states (Abaigar et al. 2001). However, functional significance of the motility of these sperm subpopulations should be profoundly investigated. Spermatozoa of cluster 1 in electroejaculated samples demonstrated some characteristics typical of hyperactivated movement in other species, in which ALH and BCF increased and VSL and LIN decreased. Only VCL in cluster 1 was lower than that reported for hyperactivated spermatozoa (Cancel et al. 2000), although the value of this parameter could be affected by the technical characteristics of the device used for the analysis. The possibility of the presence of hyperactivated spermatozoa in both electroejaculated and epididymal samples was supported by the detection of acrosomalreacted sperm in the raw semen $(6.6 \pm 5$ and $11.4 \pm 8.8 \%$ in electroejaculated and epididymal samples respectively). These data are in contrast to those reported by Yeung et al. (1996), in which the spermatozoa of the ejaculated samples that were undergoing or had completed acrosome reaction were lower in the cauda of the epididymis. However, as hyperactivated sperm motility characteristics are totally unknown in feline spermatozoa, we were unable to support this hypothesis.

To our knowledge, our study is the first to describe the kinetic characteristics of the epididymal sperm subpopulations. Semen was evaluated before (epididymal sperm) and after ejaculation (electroejaculated sperm); hence, the difference in the sperm kinetic characteristics could be due to seminal plasma or epididymal environment effects. In previous papers, in which the differences in the motility pattern between epididymal and ejaculated spermatozoa were studied (van der Horst et al. 1999, Contri et al. 2012), a heterogeneity of sperm characteristics was described.

The presence of different percentages of sperm kinetic subpopulation among the toms considered supports the individual variability of seminal sperm motility structure, as reported in other species (Nunez-Martinez et al. 2006). However, the question of how this difference affects fertility, litter size, and resistance to biotechnological (cryopreservation) or pharmacological-toxicological treatments requires further studies in the feline species.

The use of the ANNs in this study clearly showed the presence of different sperm kinetic subpopulations in both electroejaculated and epididymal spermatozoa. Similar to other species, these characteristics could reflect different functional properties of ejaculate spermatozoa. Furthermore, the discrepancies observed in electroejaculated and epididymal spermatozoa might give some information about the biological characteristics of pre- and postejaculation sperm. However, further investigation will help to clarify the biological significance of the different sperm kinetic subpopulations.

\section{Materials and Methods}

\section{Chemicals and media}

Unless otherwise stated, all reagents were purchased from Sigma Chemicals, and solutions were prepared with sterile deionized water. The medium used for dilution and washing was modified by Tyrode's HEPES-buffered medium (TALP-H; $100 \mathrm{mM} \mathrm{NaCl}, 3.1 \mathrm{mM} \mathrm{KCl}, 10.0 \mathrm{mM} \mathrm{NaHCO}, 0.3 \mathrm{mM}$ $\mathrm{NaH}_{2} \mathrm{PO}_{4}, 0.4 \mathrm{mM}$ EDTA, $21.6 \mathrm{mM}$ sodium lactate, $2.0 \mathrm{mM}$ $\mathrm{CaCl}_{2}, 0.4 \mathrm{mM} \mathrm{MgCl}_{2}, 40 \mathrm{mM}$ HEPES, and $1.0 \mathrm{mM}$ pyruvate in sterile deionized water). The $\mathrm{pH}$ was adjusted to 6.9 , and the medium was first evaluated for $\mathrm{pH}$ stability after incubation in environmental condition (temperature and air), showing no significant change in $\mathrm{pH}$. 


\section{Sperm collection}

The experimental protocol was reviewed and approved by the Health Ministry (Italy), Department of the Veterinary Public Health, Nutrition and Food Safety (Protocol number 0008366-P-04/05/2009 DSGA41014951), and the experiments were performed after obtaining consent from the owners. Seven adult (aged 2-5 years and 4-5.5 kg weight) mixed-breed toms from private owners were presented for routine orchiectomy in May. All the males were maintained under natural photoperiod conditions and provided with cat commercial dry food (Feline Adult, Hill's Science Plan) and water ad libitum.

Each tom was submitted to semen collection by electroejaculation, but sperm evaluation was not performed. After 5 days, all toms were electroejaculated again and the semen was assessed. After determination of volume and concentration, the semen was diluted in $2 \mathrm{ml} \mathrm{TALP-H}$, centrifuged ( $700 \mathrm{~g}, 6 \mathrm{~min})$, resuspended in $1 \mathrm{ml}$ of the same medium, and analyzed within $3 \mathrm{~min}$. The electroejaculation protocol used in this study was previously reported for feline semen collection (Howard et al. 1990).

After 5 days, all cats were bilaterally orchiectomized. The testes were collected in TALP-H and immediately processed. The epididymis and vasa deferentia were removed, blood vessels were completely dissected to reduce hematic contamination of semen, and the epididymal tail was separated and sliced. Spermatozoa of the epididymal tail were released into $2 \mathrm{ml} \mathrm{TALP}-\mathrm{H}$, centrifuged (700 $\mathrm{g}, 6 \mathrm{~min})$, resuspended in $1 \mathrm{ml}$ of TALP-H, and immediately analyzed.

Anesthesia for both electroejaculation and orchiectomy was achieved using $80 \mu \mathrm{g} / \mathrm{kg}$ medetomidine (Domitor, Pfizer Italia, Rome, Italy) and $5 \mathrm{mg} / \mathrm{kg}$ ketamine hydrochloride (Ketavet 100, Farmaceutici Gellini, Milan, Italy) injected intramuscularly (Johnstone 1984, Howard et al. 1990, Zambelli et al. 2007).

\section{Semen evaluation}

Each tom's electroejaculated and epididymal tail samples were observed under phase-contrast optical microscope for sperm cytoplasmic droplet detection and evaluated for motility patterns, plasma membrane integrity, morphology, and acrosome integrity. The electroejaculated semen samples were evaluated for volume and concentration. Sperm volume was determined using a variable volume pipette $(10-100 \mu \mathrm{l})$. The electroejaculated semen concentration was determined using a Bürker counting chamber (Merck) after 1:100 dilution with water. Viability, as the plasma membrane integrity, was evaluated using propidium iodide and SYBR-14 (Live/dead sperm viability kit, Molecular Probes, Inc., Eugene, OR, USA) as previously reported (Axner et al. 2002) on at least 400 spermatozoa.

Morphology evaluation was performed using fast green FCF-rose Bengal stain (Pope et al. 1991). Each slide was examined at $1000 \times$ magnification and the abnormalities concerning head, mid-piece, and flagellum were reported as percentage on at least 100 spermatozoa, while acrosomal abnormalities were considered as percentage on the total sperm acrosomes.
The acrosome membrane integrity was evaluated using FITCconjugated agglutinin derived from Pisum sativum (FITC-PSA). An aliquot of $50 \mu$ diluted semen was centrifuged at $700 \boldsymbol{g}$ for $6 \mathrm{~min}$, the supernatant was removed, and the pellet was fixed and permeabilized for at least $30 \mathrm{~min}$ at $4{ }^{\circ} \mathrm{C}$ in $95 \%$ ethanol. A droplet $(10 \mu \mathrm{l})$ of fixed semen was dried on a slide and then covered with $20 \mu$ l FITC-PSA for 10 min under dark conditions. After washing the slide thrice in bi-distilled water, $10 \mu \mathrm{l}$ mounting medium were added and a coverslip was placed on it. A total of 400 spermatozoa were analyzed with epifluorescence microscope equipped with FITC set filter. Spermatozoa with bright green fluorescence of the acrosomal region were considered to have acrosome membrane integrity, and undyed spermatozoa were regarded as the ones with reacted acrosome.

\section{Motion analysis}

Motility pattern of feline semen was evaluated using a CASA system IVOS 12.3 (Hamilton-Thorne Bioscience, Beverly, MA, USA). This device can reconstruct the trajectories of spermatozoa by the head position in frame sequences. Each setting parameter was calibrated to track feline spermatozoa and was optimized to analyze all sperms and exclude debris using the playback function. A $20 \mu \mathrm{l}$ aliquot of diluted semen was warmed for $2 \mathrm{~min}$ at $37^{\circ} \mathrm{C}$ in a water bath and $2 \mu \mathrm{l}$ diluted semen were loaded in a $20 \mu \mathrm{m}$-depth four-chamber slide (Leja, Nieuw-Vennep, The Netherlands), and ten nonconsecutive microscopic fields were analyzed.

Total motility (MOT, \%) was recorded for each sample. Furthermore, CASA system also presented the following kinetic parameters: VAP $(\mu \mathrm{m} / \mathrm{s}), \operatorname{VSL}(\mu \mathrm{m} / \mathrm{s}), \operatorname{VCL}(\mu \mathrm{m} / \mathrm{s}), \operatorname{ALH}(\mu \mathrm{m})$, BCF (Hz), STR (as VSL/VAP, \%), LIN (as VSL/VCL, \%), head elongation (ELONG, \%), and head size (SIZE, $\mu \mathrm{m})$. In addition to the computerized results, manual calculations for the WOB (as VAP/VCL, \%), DANCE (as VCL $\times A L H, \mathrm{~m}^{2} / \mathrm{s}$ ), and mean dance (MeDANCE, as ALH/LIN, \%) were carried out. All kinetic data were summarized as mean \pm s.D. for all spermatozoa of each sample and as single value for each spermatozoon.

Because of the lack of manufacturer-recommended settings for cat semen analysis, human settings were used as a model, owing to the similarities with respect to feline sperm and adjusted for cat semen analysis. In this study, 60 frames/s (Hz) and 45 frames for field settings were used.

\section{Statistical analysis}

The differences in the mean values of sperm characteristics and kinetic parameters in the electroejaculated and epididymal semen were tested using Mann-Whitney $U$ test, considering values with $P \leq 0.05$ as significant. The normality of variable distributions was evaluated by Anderson-Darling test.

The objective of this study was to identify different sperm subpopulations with specific motion characteristics in feline semen. The non-normality of distribution shapes for each kinetic parameter (Table 1) required the transformation of raw data into log-data: $y^{\prime}=\log (y+1)$, where $y^{\prime}$ is the new log-value, $y$ is the old raw value, and 1 is added to allow the evaluation of data with zero-containing variables. Multivariate analysis of 
the parameters was performed with PCA on correlation matrix with varimax rotation of the data (Systat 12, Systat Software, Inc., San Jose, CA, USA). This allowed individuating the vectors (PCs) that moved the total variability of the data and graduating them based on their importance. Thus, it was possible to obtain small independent variables owing to several characteristics with different degrees of correlation. The three most significant PCs accounted for about $80 \%$ of the variability of the sperm kinetic characteristics both in electroejaculated and epididymal spermatozoa. Loadings $\geq( \pm) 0.5$ were considered as the characteristic of that PC. For each PC pair, the densities of the spermatozoa data projected on the PC space were calculated by the kernel density technique.

Individual spermatozoa were classified into different clusters (subpopulation) based on the score for the three PCs using a neural net-based software (NeuroXL Classifier, Olsoft, Moscow, Russia). The software is a ready-to-use ANN tool integrated in the Microsoft Office Excel based on an unsupervised learning paradigm. The learning rate for the software was set to $50 \%$ of the dataset (i.e. $50 \%$ of the dataset was randomly selected for the training process), and the number of epochs, i.e. the learning iterations, was set to 1000 . This method allowed clustering of the spermatozoa (superimposed to three clusters) based on their scores on each PC.

The difference in the kinetic parameters of the subpopulations in electroejaculated and epididymal spermatozoa was estimated using Kruskal-Wallis test, followed by SteelDwass post hoc test for multiple comparisons. Differences with $P \leq 0.05$ were considered significant.

\section{Declaration of interest}

The authors declare that there is no conflict of interest that could be perceived as prejudicing the impartiality of the research reported.

\section{Funding}

This research did not receive any specific grant from any funding agency in the public, commercial or not-for-profit sector.

\section{References}

Abaigar T, Holt WV, Harrison RAP \& del Barrio G 1999 Sperm subpopulations in boar (Sus scrofa) and gazelle (Gazella dama mhorr) semen as revealed by pattern analysis of computer-assisted motility assessment. Biology of Reproduction 60 32-41. (doi:10.1095/biolreprod60.1.32)

Abaigar T, Cano M, Pickard AR \& Holt WV 2001 Use of computer-assisted sperm motility assessment and multivariate pattern analysis to characterize ejaculate quality in Mohor gazelles (Gazella dama mhorr): effects of body weight, electroejaculation technique and short-term semen storage. Reproduction 122 265-273. (doi:10.1530/rep.0.1220265)

Agatonovic-Kustrin S \& Beresford R 2000 Basic concepts of artificial neural network (ANN) modeling and its application in pharmaceutical research. Journal of Pharmaceutical and Biomedical Analysis 22 717-727. (doi:10. 1016/S0731-7085(99)00272-1)
Axner E, Pukazhenthi BS, Wildt DE, Linde-Forsberg C \& Spindler RE 2002 Creatine phosphokinase in domestic cat epididymal spermatozoa. Molecular Reproduction and Development 62 265-270. (doi:10.1002/ mrd.10070)

Barratt CLR, Tomlinson MJ \& Cooke ID 1993 Prognostic significance of computerized motility analysis for in vivo fertility. Fertility and Sterility $\mathbf{6 0}$ 520-525.

Cancel AM, Lobdell D, Mendola P \& Perreault SD 2000 Objective evaluation of hyperactivated motility in rat spermatozoa using computerassisted sperm analysis. Human Reproduction 15 1322-1328. (doi:10. 1093/humrep/15.6.1322)

Cartwright HM 2008 Artificial neural networks in biology and chemistry: the evolution of a new analytical tool. Methods in Molecular Biology 458 $1-13$.

Chantler E, Abraham-Peskir J \& Roberts C 2004 Consistent presence of two normally distributed sperm subpopulations within normozoospermic human semen: a kinematic study. International Journal of Andrology 27 350-359. (doi:10.1111/j.1365-2605.2004.00498.x)

Contri A, Valorz C, Faustini M, Wegher L \& Carluccio A 2010 Effect of semen preparation on CASA motility results in cryopreserved bull spermatozoa. Theriogenology 74 424-435. (doi:10.1016/j.theriogenology.2010.02.025)

Contri A, Gloria A, Robbe D, De Amicis I \& Carluccio A 2012 Characteristics of donkey spermatozoa along the length of the epididymis. Theriogenology 77 166-173. (doi:10.1016/j.theriogenology.2011.07.031)

van Der Horst G, Seier JV, Spinks AC \& Hendricks S 1999 The maturation of sperm motility in the epididymis and vas deferens of the vervet monkey, Cercopithecus aethiops. International Journal of Andrology 22 197-207. (doi:10.1046/j.1365-2605.1999.00171.x)

Fogel GB 2008 Computational intelligence approaches for pattern discovery in biological systems. Briefings in Bioinformatics 9 307-316. (doi:10.1093/bib/bbn021)

Holt WV, Moore HDM \& Hillier SG 1985 Computer-assisted measurement of sperm swimming speed in human semen: correlation with in vitro fertilization assays. Fertility and Sterility 44 112-119.

Holt C, Holt WV \& Moore HDM 1996 Choice of operating conditions to minimize sperm subpopulation sampling bias in the assessment of boar semen by computer-assisted semen analysis. Journal of Andrology 17 587-596.

Holt C, Holt WV, Moore HDM, Reed HCB \& Curnock RM 1997 Objectively measured boar sperm motility parameters correlate with the outcomes of on-farm inseminations: results of two fertility trials. Journal of Andrology 18 312-323.

Howard JG, Brown JL, Bush M \& Wildt DE 1990 Teratospermic and normospermic domestic cats: ejaculate traits, pituitary-gonadal hormones, and improvement of spermatozoa motility and morphology after swim up processing. Journal of Andrology 11 204-215.

Johnstone IP 1984 Electroejaculation in domestic cat. Australian Veterinary Journal 61 155-158. (doi:10.1111/j.1751-0813.1984.tb07220.x)

Krause W 1995 Computer-assisted semen analysis systems - comparison with routine evaluation and prognostic value in male-fertility and assisted reproduction. Human Reproduction 10 60-66.

Ma Y, Chen B, Wang HX, Hu K \& Huang YR 2011 Prediction of sperm retrieval in men with non-obstructive azoospermia, using the artificial neural networks: leptin is a good assistant diagnostic marker. Human Reproduction 26 294-298. (doi:10.1093/humrep/deq337)

MacLeod IC \& Irvine DS 1995 The predictive value of computer assisted semen analysis in the context of a donor insemination programme. Human Reproduction 10 580-586.

Marshburn PB, McIntire D, Carr BR \& Byrd W 1992 Spermatozoal characteristics from fresh and frozen donor semen and their correlation with fertility outcome after intrauterine insemination. Fertility and Sterility 58 179-186.

Martinez-Pastor F, Garcia-Macias V, Alvarez M, Herraez P, Anel L \& de Paz P 2005a Sperm subpopulation in Iberian Red Deer epididymal sperm and their changes through the cryopreservation process. Biology of Reproduction 72 316-327. (doi:10.1095/biolreprod.104.032730)

Martinez-Pastor F, Diaz-Corujo A, Anel E, Herraez P, Anel L \& de Paz P $2005 b$ Post mortem time and season alter subpopulation characteristics of Iberian red deer epididymal sperm. Theriogenology 64 958-974. (doi:10.1016/j.theriogenology.2005.01.003) 
Martinez-Pastor F, Tizado EJ, Garde IJ, Anel L \& de Paz P 2011 Statistical series: opportunities and challenges of sperm motility subpopulation analysis. Theriogenology 75 783-795. (doi:10.1016/j.theriogenology. 2010.11.034)

McCulloch WS \& Pitts W 1943 A logical calculus of the ideas imminent in nervous activity. Bulletin of Mathematical Biology 5 115-133.

Mortimer ST \& Swan MA 1995 Kinematics of capacitating human spermatozoa analysed at $60 \mathrm{~Hz}$. Human Reproduction 10 873-879.

Muino R, Rivera MM, Rigau T, Rodriguez-Gil JE \& Pena AI 2008 Effect of different thawing rates on post-thaw sperm viability, kinematic parameters and motile sperm subpopulations structure of bull semen Animal Reproduction Science 109 50-64. (doi:10.1016/j.anireprosci. 2007.11.028)

Nunez-Martinez I, Moran JM \& Pena FJ 2005 Do computer assisted derived morphometric sperm characteristics reflect DNA status in canice spermatozoa? Reproduction in Domestic Animal 40 537-543. (doi:10. 1111/j.1439-0531.2005.00628.x)

Nunez-Martinez I, Moran JM \& Pena FJ 2006 A three-step statistical procedure to identify sperm kinematic subpopulations in canine ejaculates: change after cryopreservation. Reproduction in Domestic Animal 41 408-415. (doi:10.1111/j.1439-0531.2006.00685.x)

Perez-Llano B, Yenes-Garcia P \& Garcia-Casado P 2003 Four subpopulation of boar spermatozoa defined according to their response to the short hypoosmotic swelling test and acrosome status during incubation at $37^{\circ} \mathrm{C}$. Theriogenology 60 1401-1407. (doi:10.1016/ S0093-691X(03)00131-6)

Pope CE, Zhang YZ \& Dresser BL 1991 A simple staining method for evaluating acrosomal status of cat spermatozoa. Journal of Zoo and Wildlife Medicine 22 87-95.

Quintero-Moreno A, Mirò J, Rigau T \& Rodriguez-Gil JE 2003 Identification of sperm subpopulations with specific motility characteristics in stallion ejaculates. Theriogenology 59 1973-1990. (doi:10.1016/S0093691X(02)01297-9)

Quintero-Moreno A, Rigau T \& Rodriguez-Gil JE 2004 Regression analyses and motile sperm subpopulation structure study as improving tools in boar semen quality analysis. Theriogenology 61 673-690. (doi:10.1016/ S0093-691X(03)00248-6)
Quintero-Moreno A, Rigau T \& Rodriguez-Gil JE 2007 Multivariate cluster analysis regression procedures as tools to identify motile sperm subpopulation in rabbit semen and to predict semen fertility and litter size. Reproduction in Domestic Animal 42 312-319. (doi:10.1111/j. 1439-0531.2006.00785.x)

Ramesh AN, Kambhampati C, Monson JR \& Drew PJ 2004 Artificial intelligence in medicine. Annals of the Royal College of Surgeons of England 86 334-338. (doi:10.1308/147870804290)

Rijsselaere T, Van Soom A, Maes D \& de Kruif A 2003 Effect of technical settings on canine semen motility parameters measured by the HamiltonThorne analyzer. Theriogenology 60 1553-1568. (doi:10.1016/S0093691X(03)00171-7)

Roldan ERS 1998 Signal transduction during mammalian sperm acrosomal exocytosis. In Gametes: Development and Function, pp 219-228. Eds A Lauria, F Gandolfi, G Enne \& L Gianaroli. Rome: Serono Symposia.

Samli MM \& Dogan I 2004 An artificial neural network for predicting the presence of spermatozoa in the testes of men with nonobstructive azoospermia. Journal of Urology 171 2354-2357. (doi:10.1097/01.ju. 0000125272.03182.c3)

Yeung CH, Cooper TG \& Weinbauer GF 1996 Maturation of monkey spermatozoa in the epididymis with respect to their ability to undergo the acrosome reaction. Journal of Andrology 17 427-432.

Zambelli D \& Cunto M 2006 Semen collection in cats: techniques and analysis. Theriogenology 66 159-165. (doi:10.1016/j.theriogenology. 2006.01.054)

Zambelli D, Cunto M, Prati F \& Merlo B 2007 Effects of ketamine or medetomidine administration on quality of electroejaculated sperm and on sperm flow in the domestic cat. Theriogenology 68 796-803. (doi:10. 1016/j.theriogenology.2007.06.008)

Received 8 April 2012

First decision 2 May 2012

Revised manuscript received 5 June 2012

Accepted 29 June 2012 